\title{
Analogics and its Cognitive Efficiency
}

Joonho KIM

Department of International Relations

Tokyo International University

Matoba 2509, Kawagoe, Saitama, Japan

joonho@tiu.ac.jp

ABSTRACT: Analogy is a logical methodology, which can maximize the efficiency of human reasoning. Notwithstanding, few papers analyzed its logical characteristic. In this paper it is claimed that analogy is a typical form of logics, and the term and concept "analogics" is suggested. The reasoning principle, structure and process of this logicis are shown with some examples. It is certain that the analogics has great cognitive efficiency but it must obey rules to acquire the logical validity.

Keywords: Logic, Cognition

Received: 23 October 2018, Revised 4 January 2019, Accepted 19 January 2019

(C) 2019 DLINE. All Rights Reserved

DOI: $10.6025 / \mathrm{jcl} / 2019 / 10 / 2 / 50-56$

\section{Introduction}

Analogy is a cognitive method of acquiring knowledge by comparing two objects. It is an interpretation of an object, based upon things familiar to the observer. This reasoning stands on the supposition that the causality or the function of one thing can be recognized in reference to the other thing.

This is quite an efficient logic system, in that the unknown object can be recognizable only by expanding or reusing the known. The habitability of Mars, about which the observatory information is insufficient, is surmised by the geological conditions of the earth. And the principle of our solar system is used to explain planetary systems in many other galaxies.

The cognitive function of analogy is to bridge the known and the unknown, the epistemological terra incognita ${ }^{1}$.

This cognitive methodology is a logic, for it gives us a certain inferential process and the conclusion but its logical character is different from deduction and induction, although its logical structure is the composite of the two.

Logic is the structure of statements on the truth, and a statement in essence is a movement from the premise $(X)$ to the conclusion $(Y)$ :

$$
X \rightarrow Y
$$


Here induction means, in its nature, a judgment from the particular to the general. Deduction is from the general to the particular. But analogics, which is analogy as a logic, is a movement from one particular to the other particular (see Table 1).

So analogics is quite a peculiar type of logic, and it has its own inference rule, which belongs not to any of former logics.

\begin{tabular}{|l|c|c|}
\hline \multirow{2}{*}{ Induction } & $\mathrm{X}$ & $\mathrm{Y}$ \\
\cline { 2 - 3 } & particular & general \\
\hline Deduction & general & particular \\
\hline Analogics & particular & particular \\
\hline
\end{tabular}

Table 1. Characteristics of logics

How can one particularity be understood by the other particular?

Basically it is impossible. A particularity has no right to define other particulars. Therefore analogics 2 should have universal principle in its background, which functions as the device to translate one particular to the other.

\section{The Reasoning of Analogics}

\subsection{Logical Process}

If analogics presupposes the universal truth as the criteria, then we can draw the reasoning as below. Let's think of a logic vector. Let an expression.

$X \rightarrow A$ denote inductive process, which has the movement from the particular to the general. And $A \rightarrow Y$ is the deductive process, which has the movement from the general to the particular. And finally, this process is concluded as $X \rightarrow Y$, which seems to show the movement from the particular to the particular.

This process is drawn in Figure 1.

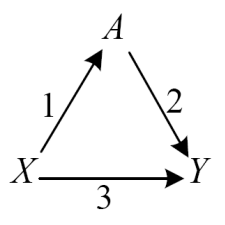

Figure 1. The logic triangle

These logic vectors in the triangle are written as below: $X_{\rightarrow}^{1} A \stackrel{2}{\rightarrow} Y=X \stackrel{3}{\rightarrow} Y$

Here, analogical process includes the intermediate element of $A$, even though it looks like $X \rightarrow Y$.

${ }^{1}$ Some authors use the terms "source or base" and "target" domain, but the latter looks like a strong teleological concept. "Known" and "unknown" seem to be simple and give us little misunderstanding.

${ }^{2}$ The Greek word "logos" is divided into two suffixes: "-logy" and "-logic" or "-logism". The former is usually used in academic disciplines, and the latters seem to remind of logics. Here I suggest new terminology "analogics" to emphasize the logical aspects of analogy, which has the composite logical characters in the background, as well as to evade the confusion when using "analogic", the adjective form of analogy. 
Logic lines 1 and 2 is not the sufficient condition for the logic line 3, but they show there are composite logics behind the seemingly simple analogical line 3 .

This is the basic structure of analogics. Here the mapping is not done directly between $X$ and $Y$, but $X$ is reflected via $A$ on $Y$.

Suppose $X$ is the known object, and $Y$ is the unknown object.

Analogy matches the unknown elements in $Y$ inferentially to the elements in $X$ 's structure. Then analogy means to correspond the elements of $X$ to $Y$, and form the structure in $Y$, based on $X$ as the known system model.

The objects consist of special elements and common elements; common general truth and unique characters peculiar to each object. These characters of the common and the unique form the reality of each object.

Two entities $X, Y$ are defined as below, with their elements $a, b, c$ :

$$
\begin{aligned}
& X=a+b \\
& Y=a+c
\end{aligned}
$$

Here $a$ is the common part, $b, c$ are particular parts which are unique to $X$ and $Y$. The elements $a, b$ are known, $c$ is unknown.

Analogical reasoning need not be precise in every detail.

So the similarity of $X, Y$ is written as fuzzy identity, as below

$$
X \cong Y
$$

This is described with each parts of $X$ and $Y$ as

$$
(a+b) \cong(a+c)
$$

Here $X, Y$ are not the identical, but approximately same.

All these processes are concluded as below,

$$
\therefore b \cong c
$$

The uniqueness of $X$ and $Y$ is discarded, and common characteristics are corresponded, so the logical generality becomes possible. Therefore, the unknown $c$ is approximately confirmed by the known $b$.

\subsection{Mapping in the Holistic Structure}

Analogy is a cognitive activity based upon the correspondence of elements in two objects, and so the mapping is essential. And structure mapping theory can be said to have been the starting point to theorize the analogy (refer [3] as a basic literature).

Is it able to explain the reality of $Y$, using the principles derived from the $X$ 's reality?

Analogical comparison is based upon the similarity of the structure. The structure is the composition and the organization of elements, and it is the holistic relation of the elements within an object. This may be called the holistic individuality. The entity is disassembled into parts, and the parts are compared and correspond to the parts of other object, and the unknown parts are fixed.

An analogy is possible, because every element is related to others as a part of the whole structure. The fact that the possibility of analogy lies in that each entity is a whole in its structure, can be called the condition of holistic entity, in analogics. 
Structure is the relation between the whole and the part, and the characteristic is the relation between the parts. If it were not for the wholeness, there should be no meaning or the relation of the elements in the two.

\section{Analogical Examples}

Analogics is used in many parts of academic and pragmatic fields. Problem solving, learning, argumentation, understanding metaphor, scientific theory formation, explanation and case-based reasoning [7, p.65], etc. Several examples are suggested below to explain the logical characteristics of the analogics.

All these examples are classified into ontological type (as problem-solving) or epistemological type (comparison and understanding as in parables), and also they are intertwined (the political adaptation of fables).

\subsection{Analogy of Physics}

The physical structure and law which is described below as the relation between elements and their causalities, shows clearly the analogical correspondence, in which similar structures share similar functions.

These structures belong to different physical worlds, but their characteristics as flow share the principles in common. One is the Poiseuille's Law of water flow, and the other is Ohm's Law of electric current.

Flow and current are in essence the same, and the related concepts are structurally similar and correspond to each element of the laws:

a. Poiseuille's Law: $F=\Delta P / R$

b. Ohm's Law: $I=\Delta V / R$

In water flow system (a), $F$ is flow rate, $\Delta P$ is pressure difference, and $R$ is resistance.

In electric circuit (b), $I$ is current, $\Delta V$ is potential difference, and $R$ is resistance $[4, \mathrm{p} .10]$.

These two laws structure mapping theory, which shows the exact correspondence of the elements:

$$
F: I, \Delta P: \Delta V, R: R
$$

One to one correspondence of elements and overall similarity between the two structures links the analogical functionality in each system.

The experience "the structure of an example determines the function of the example" is encoded in a connection [structure, function]. Often this connection is not given explicitly for all examples. But, from the connection [structure, function] one can infer analogically that if two examples, base and target, are similar with respect to their structure then they may correspond with respect to their function too $[5, \mathrm{p} .10] \ldots$

Likewise, atom structure in physics and solar system structure in astronomy are similar to each other and have similar functions.

\subsection{Parables and Fables}

A parable is "an earthly story with a heavenly meaning" [1, p.12].

Parable explains the metaphysical religious truth, using the daily episodes. It has analogical reasoning between the truth and the story.

A parable is a similitude or full-length story, true to nature and to life, a picture of something which can be observed in the world of our experience, which was told by our Lord to illustrate a divine truth [2, p. 5].

The Christian parables like the good Samaritan, the lost sheep and the prodigal son have the structure, philosophy, value,

International Journal of Computational Linguistics Research Volume 10 Number 2 June 2019 
elements, lesson.

Even between religions this similarity of structures is revealed.

"The poor woman's lamp" in Buddhism which is the counterpart of Christian "the widow's mite" is a quasiparable about a poor woman who dedicated most of her money to worship Buddha.

Mathematically the moral of it is interpreted like below; for example, when the poor woman served 1 lamp spending 1 rupee out of 2 rupees which was all what she had, then she spent $50 \%$ of all her property. On the other hand, a king or a rich man served 1000 lamps spending 1000 rupees out of 1,000,000 rupees, then it is $0.1 \%$ of the property. The fact is that $50 \%$ spending is more than $0.1 \%$, and the poor woman sacrifices 500 times as much as the king or the rich man.

It goes without saying that a parable with the story is easier and softer to understand than mathematics.

Fables have the same effects as religious parables. The goose with the golden egg, the sun and the north wind, the hare and the tortoise and the ant and the grasshopper are famous titles of Aesop's fables.

They are generally animal stories with human wisdoms, which are experienced mainly in nature world.

\subsection{Problem Solving}

Heuristic adaptation of analogy is a creative activity in problem solving.

Let's think of a mountain the height of which is $A B$ (Figure 2). Suppose $B E, C D, D E$ are known, but $A B$ is unknown. The structures of $\mathrm{f} \triangle A B E$ and $\triangle C D E$ define each elements proportionately. By the similarity of the two triangles, one can easily solve the problem of measuring the height of the mountain.

There is a unique case, in which a fable is linked to political problem solving.

The Korean peninsula has been divided between South and North, in Cold War era, and the two sides have confronted more than half a century with each other.

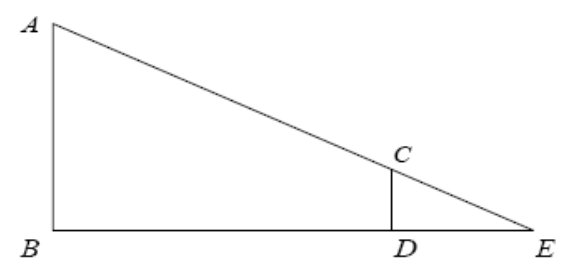

Figure 2. An example of heuristic problem solving

At the end of 1990s, the government of South Korea announced a peace plan named "Sunshine policy", which explicitly came from Aesop's fable, "the sun and the north wind". The government of South Korea felt the limitation and risk of physical force and decided to offer economic aids and other supports to catch the North's heart. Several years later, the leaders of South and North had the meeting for the first time in history.

This policy imitated the Aesop's fable. Here the real policy and the fable have structural similarity. Fable's moral was transformed into political strategy. And fable simile is linked to physical effect.

\subsection{Legal Analogy}

It is impossible to stipulate all the judiciary cases in society. So that, in law, a known rule is often used to interpret unknown, similar cases.

Since the number of legal rules is restricted and their content is often incomplete, it is necessary at times for a lawyer to opt for 
an analogical application of a legal rule to a given case in order to decide the case properly.

Legal analogy is conceived as a process of generation of hypothetical rule which supplies the lack of law for a certain particular case $[8$, p.110].

Here we can understand that analogy is not only efficient, but also essential to the judiciary judgment, because it is practically the only way to respond to the innumerable particular cases in daily lives.

The process of analogical thought in law appears to work in four simple steps: (1) Some fact pattern $A$ has a certain characteristic $\mathrm{X}$, or characteristics $\mathrm{X}, \mathrm{Y}$ and $\mathrm{Z}$; (2) Fact pattern B differs from A in some respects but shares characteristics X, or characteristics X, Y, and Z; (3) The law treats A in a certain way; (4) Because B shares certain characteristics with A, the law should treat $B$ the same way[6, p.745].

The reasoning similar to the typical analogical process is found here.

\section{The Effect and Defect of Analogics}

Analogics produces logical efficiency, which is to easily fix an uncertain object, according to a certain, perceivable, cognitive structure of the known object.

The positivity of analogy lies in the functional significance of economies of research, for example. The process, conclusion, concepts and principles are derived from other disciplines, and therefore an academic result of a discipline can be reproduced with minimum cost, One good analogy is worth three hours' discussion (Dudley Field Malone) ${ }^{3}$, because cognitive agents can learn a new conceptualization of the target domain without perceiving a huge number of examples [4, p.10].

Analogical reasoning may be a second-best alternative to the search for reflective equilibrium in light of the multiple constraints imposed on any legal system in the real world.

First, reasoning by analogy may be the best approach available for people of limited time and capacities. Second, reasoning by analogy may have the significant advantage of allowing people unable to reach anything like an accord on general principles to agree on particular outcomes.

Third, analogical reasoning may be especially desirable in contexts in which we seek moral evolution over time.

Fourth, analogical reasoning in law operates with precedents that have the status of fixed points [6, p.782].

But the problem is that the easy adaptation of this methodology may produce error. Economies of analogy are likely to fall into the fallacy of methodological convenience, and are misused as a tool of expediency. Common parts may be analogic through the correspondence of concepts and elements, while the particular parts are not necessarily correspondent, so that misinterpretation may occur.

And the similarity may be varied according to the subjectivity of the observer, that is, it includes certain points of view, so that there exists no absolute objective similarity between things.

So there have been objections against analogy, as below:

1. Absence of scientific, external or critical perspectives,

2. Indeterminacy; dependence on consensus,

${ }^{3}$ http://www.brainyquote.com/quotes/quotes/d/dudleyfiel119362.html

International Journal of Computational Linguistics Research Volume 10 Number 2 June 2019 
3. The search for relevant differences-the inevitable need for criteria never supplied by analogical reasoning [6, pp.767- 773].

Analogics is a logic with the possibility of practical conclusion, which stands as long as the empirical validity of fuzzy judgment, which is not the precise reasoning, is not denied.

Analogics expands the range of reasoning largely by the fuzzy equalization. This direct correspondence saves the transformation cost, and brings out the conclusion with ease.

Behind the criticism on the analogic method hides the fallacy of generalization. Analogics is a logical method which interprets a particular $Y$ based on the other particular $X$. Superficial similarity incurs the statement $Y$ because of $X$. Here the possibility of the fallacy of generalization occurs, which begets the risk of mapping of from a particular to a particular. It is a serious defect for the logic, because a particular $X$ may be misunderstood as a general criterium, although a particular itself cannot be an absolute criterium. Criteria exist beyond particulars $X, Y$, which guarantees the right judgment.

What counts is not so much the similarity of attributes as the commonality of the structure. This point of view can overcome the fallacy.

The logic of analogy becomes a 'logic' of the justification of analogy and the use of an analogy is reduced to the use of an analogy in the justification of a new hypothesis or solution [7, p. 67]. And here analogics is suggested as the negation of the logic of analogy.

\section{Conclusion}

About 2,500 years ago, there lived a beauty named Xi Shi(西施) in China, who looked pretty even when she frowned. An ugly girl, seeing that Xi Shi frowned and looked pretty, made up her mind to imitate Xi Shi, hoping that she looked pretty too. But on seeing the ugly girl frown, all villagers ran away from her. This story is called “the mimicry of frowning (效馨)". There exists no general logic of aesthetics which links Xi Shi and the ugly girl, so that the causality of frowning and cuteness of Xi Shi is not probable in the case of the ugly girl. Between these two girls parallels the peculiarities which cannot be generalized.

The attempt to squeeze out the conclusion with ease by adapting the analogy automatically, even in the situation where the particularity, without shared generality, collides with each other, is sure to reproduce the tragedy of the ugly girl, who misjudged the frowning as the sufficient condition to efficiently and easily acquire attractiveness.

\section{References}

[1] Barclay, W. (1970). And Jesus said. The Westminster Press.

[2] Doerksen, V. (1970). The interpretation of parables. Grace Theological Journal. 11-2.

[3] Gentner, D. (1983). Structure-mapping: A theoretical framework for analogy. Cognitive Science. Vol. 7.

[4] Gust, H., Krumnack, U., Kühnberger, K-U., Schwering, A. (2008). Analogical reasoning: a core of cognition. KI Zeitschrift Künstliche Intelligenz, 1/08.2008.

[5] Kerber, M., Melis, E., Siekmann. J. (1992). Analogical reasoning with typical examples. SEKI Report. SR-92-13.

[6] Sunstein, C. (1993). On analogical reasoning. Harvard Law Review. 106 (3).

[7] Van Dormael J. (1990). The emergence of analogy: analogical reasoning as a constraint satisfaction process. Philosophica. Vol. 46. Ghent University.

[8] Yoshino, H., Haraguchi, M., Sakurai, S., Kagayama, S. (1993). Towards a legal analogical reasoning system: knowledge representation and reasoning methods. TheFourth International Conference on Artificial Intelligence and Law. The Association for Computing Machinery. 